\title{
DIAMETRAL CONTRACTIVE MAPPINGS IN REFLEXIVE BANACH SPACES
}

\author{
Milan R. Tasković*
}

\begin{abstract}
In this paper it is proved that if $K$ is a nonempty bounded closed convex subset of a reflexive Banach space $X$ and if $K$ has normal structure, then any diametral contractive mapping $T$ on $K$ into itself has a fixed point.
\end{abstract}

\section{Introduction and history}

Fixed-point theorems for families of continuous linear or affine transformations have been obtained by Markoff [15], Kakutani [10], Day [5] and Göhde [7]. Fixed-point theorems for families of nonexpansive mappings established by De Marr [6] (for Banach spaces), F. Browder [3] (for uniformly convex spaces), and T. Lim [14] (for reflexive Banach spaces).

Let $X$ be a Banach space. A mapping $T$ of a subset $K$ of $X$ into $X$ is called diametral contraction on $K$ if

$$
\|T x-T y\| \leq \sup \{\|x-z\|: z \in K\}
$$

for all $x, y \in K$. In [21] we investigated a nonempty commutative family $\mathcal{F}$ of diametral contractive mappings of a nonempty compact convex subset $K$ into itself, and showed that the family $\mathcal{F}$ has a common fixed point in $K$.

The existence of fixed points of a nonexpansive mapping on a Hilbert space was first established by Browder [3], and later by Browder [4], Göhde [8] and Kirk [13] for such mappings on uniformly convex Banach spaces, indipendently of each other.

On the other hand, a general fixed point result for isometries was obtained by Brodskij-Milman [2]. Later, Kirk [13] proved that if $K$ is a nonempty weakly compact convex subset of a Banach space and if $K$ has normal structure, then every nonexpansive self-map $T$ of $K$ has a fixed point.

AMS (MOS) Subject Classification 1991. Primary: 47H10, 05A15. Secondary: 54H25.

Key words and phrases: Reflexive Banach spaces, normal structure, Šmulian property, Browder-Göhde-Kirk theorem, diametral contractive mappings, fixed points.

*Research supported by Science Fund of Serbia under Grant 1457. 
In this paper we extend this Kirk's theorem and we describe a result for the existence of fixed points which generalize several known results.

\section{Main result}

For $S \subset X$ we denote the diametar of $S$ by $\delta(S)$. A point $x \in S$ is a diametral point of $S$ provided

$$
\sup \{\|x-y\|: y \in S\}=\delta(S) .
$$

A convex set $K \subset X$ is said to have normal structure if for each bounded convex subset $H$ of $K$ which contains more than one point, there is some point $x \in H$ which is not a diametral point of $H$. Compact convex sets, possesses normal structure obtained by Brodskij-Milman [2]. In this paper the concept of normal structure, due to Brodskij-Milman [2], plays a key role.

We are now in the position of proving the following statement use a characterization of reflexivity due to Šmulian.

Theorem 1. Let $K$ be a nonempty, bounded, closed and convex subset of a reflexive Banach space $X$, and suppose that $K$ has normal structure. If $T$ is a diametral contractive mapping of $K$ into itself, then $T$ has a fixed point in $K$.

In the proof of this result we shall make use the following well-known statement.

Theorem 2. (Šmulian [19]). A necessary and sufficient condition that a Banach space $X$ be reflexive is that every bounded descending sequence (transfinite) of nonempty closed convex subsets of $X$ has a nonempty intersection.

Proof of Theorem 1. Let $\mathcal{G}$ denote the collection of all nonempty closed and convex subsets of $K$, each of which is mapped into itself by $T$. Obviously, $\mathcal{G}$ is nonempty. Now we define a partial ordering on $\mathcal{G}$ by the set inclusion relation. By Šmulian's theorem and Zorn's lemma $\mathcal{G}$ has a minimal element which we denote by $S$. Here, $S$ being minimal with the respect to being nonempty, bounded, closed, convex and invariant under $T$. We complete the proof by showing that $S$ consists of a single point.

If $S$ contains only one element, then that element is a fixed point of $T$. If not, let $S$ contain more than one element, then it follows from the normal structure of $K$ that there exists $c \in S$ such that

$$
\sup \{\|c-y\|: y \in S\}:=\rho<\delta(S) .
$$

Thus and from (D) we obtain the following inequality

$$
\|T c-T y\| \leq \sup \{\|c-y\|: y \in S\}=\rho .
$$


Hence, $T(S)$ is contained in the closed ball $B$ with $T c$ as center and $\rho$ as radius. Also, $S \cap B$ is invariant under $T$, therefore, by the minimality of $S$ it follows that $S \subset M$, i.e., $\|T c-x\| \leq \rho$ for every $x \in S$. Hence, we have

$$
\sup \{\|T c-x\|: x \in S\} \leq \rho .
$$

Consider the set

$$
F=\{z \in S: \sup [\|z-x\|: x \in S] \leq \rho\} .
$$

Obviously $F$ is closed, convex and nonempty $(T c \in F)$. Again if $z \in F$, then $z \in S$ and hence $T z \in F$ by (1). Hence $F$ is invariant under $T$. Also, we have

$$
\delta(F) \leq \sup \{\|x-y\|: x \in F, y \in S\} \leq \rho<\delta(S),
$$

by hypothesis and the preceding facts. Thus $F$ is a proper subset of $S$, which contradicts the minimality of $S$. Hence $S$ has only one element which is a fixed point of $T$. The proof is complete.

\section{Some consequences}

This section gives applications of the preceding result. Many results which are closely related to results on uniformly convex spaces have been published recently. In this sense we give the following essential result.

Corollary 1. (Uniformly convex spaces). Let $X$ be a uniformly convex Banach space and $K$ a nonempty bounded closed convex subset of $X$, and $T$ be a self-map of $K$. If there are real numbers $a, b, c \geq 0$ such that

$$
\|T x-T y\| \leq a\|x-y\|+b\|x-T x\|+c\|x-T y\|
$$

and $a+b+c=1$ for all $x, y \in K$, then $T$ has a fixed point in $K$.

Special cases of Corollary 1 have been disscussed by Roux-Soardi [17] (for $c=0$ ), Browder-Göhde-Kirk [4, 8, 13] (for $b=c=0$ ), Reich [16] (for $c=0$ ) and Soardi [18] (for $b=c=0$ ).

Since the reflexivity of the space and the normal structure of $K$ being consequences of the uniform convexity of $X$, we obtain the preceding results as directly consequences of Theorem 1 .

Proof of Corollary 1. From the inequality (G) we have the following inequalities

$$
\begin{gathered}
\|T x-T y\| \leq a\|x-y\|+b\|x-T x\|+c\|x-T y\| \leq \\
\leq(a+b+c) \sup \{\|x-y\|: y \in K\}=\sup \{\|x-y\|: y \in K\},
\end{gathered}
$$

i.e., we obtain that $(\mathrm{G})$ implies $(\mathrm{D})$ in Theorem 1 . Hence and from the preceding facts $X$ and $T$ satisfy all the required hypotheses in Theorem 1. Applying Theorem 1 we obtain that $T$ has a fixed point in $K$. The proof is complete.

We notice, by interchanging $x$ and $y$ in $(\mathrm{G})$, from Theorem 1 we obtain directly the following result. 
Corollary 2. Let $K$ be a nonempty bounded, closed and convex subset of a reflexive Banach space $X$ and suppose that $K$ has normal structure. If $T$ maps $K$ into itself and if there are real numbers $a, b, c \geq 0$ such that

$$
\begin{gathered}
\|T x-T y\|-a\|x-y\| \leq \\
\leq \max \{b\|x-T x\|+c\|x-T y\|, b\|y-T y\|+c\|y-T x\|\}
\end{gathered}
$$

and $a+b+c=1$ for all $x, y \in K$, then $T$ has a fixed point in $K$.

As an immediate consequence of Theorem 1 we obtain the following statement.

Corollary 3. (Tasković [21]). Let $K$ be a nonempty, bounded, closed and convex subset of a reflexive Banach space $X$, and suppose that $K$ has normal structure. If $T$ is a mapping of $K$ into itself such that

$$
\sup \{\|T x-T y\|: y \in K\} \leq \sup \{\|x-y\|: y \in K\}
$$

for every $x \in K$, then $T$ has at least one fixed point in $K$.

An annotation. This result, for the class (A) of mappings $T: K \rightarrow K$, is a special case of the more general result for the class (D) obtained in 1980 by Tasković [21].

In connection with this, we notice that this result has been again consider in 1983, three year next, from Jaggi (for this see: D.S. Jaggi, On fixed points of nonexpansive mappings, Contemporary Math., 21 (1983), 147-149.).

\section{An open problem}

It is natural to ask whether the preceding Theorem 1 remains true for reflexive Banach spaces without normal structure.

First characterization of normed spaces with normal structure was given by Brodskij-Milman [2] in terms of diametral sequences.

A bounded sequence $\left\{x_{n}\right\}_{n \in \mathbb{N}}$ is diametral if it is non-constant and if $\delta\left(x_{n}, \operatorname{conv}\left\{x_{1}, \ldots, x_{n-1}\right\}\right) \rightarrow \delta\left(\left\{x_{n}\right\}_{n \in \mathbb{N}}\right)$, where conv $A=$ convex hull of $A$.

Let $X$ be a normed space and we define $\mathcal{K}$ as a collection of all closed, convex, bounded subsets of $X$, each of them containing more than one element.

One says that $X$ has normal structure if every $K \in \mathcal{K}$ contains a point which is not diametral point of $K$.

In connection with the preceding, a mapping $T: K \rightarrow K$ (for $K \in \mathcal{K}$ ) is said to be diametral contractive if

$$
\|T x-T y\| \leq \sup \{\|x-z\|: z \in Y\}
$$


for every $Y \in \mathcal{K}$ with $Y \subset K$ and for all $x, y \in Y$. A normed space $X$ is said to have diametral fixed point property if every diametral contractive map $T: K \rightarrow K$ (for an arbitrary $K \in \mathcal{K}$ ) has a fixed point.

If every normed space $X$ have diametral fixed point property does $X$ has normal structure?

\section{References}

[1] L.P. Belluce and W.A. Kirk: Nonexpansive mappings and fixed-points in Banach spaces, Illinois J.Math., 11(1967), 474-479.

[2] M.S. Brodskij and D.P. Milman: On the center of a convex set, Dokl. Akad. Nauk SSSR (N.S.), 59 (1948), 837-840.

[3] F.E. Browder: Fixed point theorems for noncompact mappings in Hilbert space, Proc. Nat. Acad.Sci. U.S.A., 53 (1965), 1272-1276.

[4] F.E. Browder: Nonexpansive nonlinear operators in a Banach space, Proc. Nat. Acad. Sci., 54 (1965), 1041-1044.

[5] M.M. Day: Fixed-point theorems for compact convex sets, J. Math., 5 (1961), 585-590.

[6] R. De Marr: Common fixed-points for commuting contraction mappings, Pacific J. Math., 13 (1963), 1139-1141.

[7] D. Göhde: Über Fixpunkte bei stetigen Selbstabbildungen mit kompakten Iterierten, Math. Nach., 28 (1964), 45-55.

[8] D. Göhde: Zum Princip der kontraktiven Abbildung, Math. Nachr., 30 (1965), 251-258.

[9] K. Goebel, W.A. Kirk and T.N. Shimi: A fixed point theorem in uniformly convex spaces, Bollettino U.M.I., 7 (1973), 67-75.

[10] S. Kakutani: Two fixed-point theorems concerning bicompact convex sets, Proc. Imp. Acad. Tokyo, 14 (1938), 242-245.

[11] R. Kannan: Some results on fixed points III, Fund.Math., 70 (1971), 169-177.

[12] M. A. Khamsi, and D. Misane: Compactness and covexity structures in metric spaces, Math.Japonica, 41(1995), 321-326.

[13] W.A. Kirk: A fixed point theorem for mappings which do not increase distances, Amer. Math. Monthly, 72 (1965), 1004-1006.

[14] T.C. Lim: A fixed point theorem for families of nonexpansive mappings, Pacific J. Math., 53(1974), 487-493.

[15] A. Markoff: Quelques théorémes sur les esembles Abèliens, Doklady Akad. Nauk SSSR (N.S.), 10 (1936), 311-314. 
[16] S. Reich: Kannan's fixed point theorem, Boll.Un.Mat. Ital., 4 (1971), 1-11.

[17] D. Roux and R. Soardi: Alcune generalizazioni del Teorema di Browder-GöhdeKirk, Lincei-Rend. Sc.fis.mat.e nat., 52 (1972), 682-688.

[18] R. Soardi: Su un problema di punto unito di S. Reich, Boll.Un.Mat. Ital., 4 (1971), 841-845.

[19] V. Šmulian: On the principle of inclusion in the space of type (B), Math. Sb. (N.S.), 5 (1939), 327-328.

[20] M.R. Tasković: Reflexive Banach space and fixed point theorems, Publ. Inst. Math. (Beograd), 34 (1976), 243-247.

[21] M.R. Tasković: Some results in the fixed point theory-II, Pub. Inst. Math. (Beograd), 41 (1980), 249-258.

Matematički fakultet 11000 Beograd, P.O. Box 550 Yugoslavia Received January 7, 2000. 